\title{
APPROXIMATING THE INVARIANT DENSITIES OF TRANSFORMATIONS WITH INFINITELY MANY PIECES ON THE INTERVAL
}

\author{
P. GÓRA AND A. BOYARSKY
}

(Communicated by Kenneth R. Meyer)

\begin{abstract}
Let $I=[0,1]$ and $\tau: I \rightarrow I$ be a piecewise continuous, expanding transformation with infinitely many pieces of monotonicity. We construct a sequence of transformations $\left\{\tau_{n}\right\}$, each having a finite partition, such that their invariant densities converge in $L_{1}$ to the invariant density of $\tau$.
\end{abstract}

\section{INTRODUCTION}

Let $I=[0,1]$ and let $\tau: I \rightarrow I$ be a piecewise continuous, expanding transformation with finite of infinitely many pieces of monotonicity. When $\tau$ admits an absolutely continuous invariant measure (acim) $\mu$, with density function $f$, we are interested in a method for approximating $f$. If $\tau$ has a finite number of pieces, results are available [3,5]. In [4], it is shown that piecewise linear Markov maps $\left\{\tau_{n}\right\}$ can be constructed which approach $\tau$ uniformly and whose densities $\left\{f_{n}\right\}$ converge in $L_{1}$ to $f$. This is a strong result which cannot be derived from [3] since the essential inequality there involves a constant which depends inversely on the minimum length of the partition.

There are important transformations which have an infinite number of pieces, for example, the Gauss transformation $\tau(x)=1 / x(\bmod 1)$. Also, first return maps for any map $\tau$ give rise naturally to transformations with a countable number of pieces [2]. There are no known approximation theorems that apply to such maps. However, the method of this note will allow the approximation of the density for such $\tau$.

Further motivation for approximating transformations with infinite pieces comes from computer implementations of dynamical systems. Suppose $\tau$ has a countable number of pieces. Then any computer representation, $\hat{\tau}$, of $\tau$ will necessarily have only a finite number of pieces. This invites the question: will the absolutely continuous measure associated with $\hat{\tau}$ be close to the acim of $\tau$ ? This problem was studied in [7] in the case when $\tau$ has a finite partition. With

Received by the editors July 31, 1988.

1980 Mathematics Subject Classification (1985 Revision). Primary 28D05; Secondary 58F11.

The research of the second author was supported by NSERC and FCAR grants. 
the results of this note, the invariant density of a map with infinite number of pieces can be approximated by the invariant density of a map with finite number of pieces, $\tau_{n}$, and by the results of [7], the computer representation of $\tau_{n}, \hat{\tau}_{n}$, has associated with it an absolutely continuous measure which approximates the invariant density of $\tau_{n}$. This result is of interest since it proves that histograms of computer orbits $\left\{\hat{\tau}_{n}^{i}(x)\right\}_{i=1}^{\infty}$ can be used to approximate invariant density of the map $\tau$ with an infinite number of pieces.

Using a key lemma from [1], a compactness result analogous to that proved in [4] is established in $\S 2$ for families of transformations with a countable number of pieces. This is used to prove the following approximation theorem: the invariant density of a piecewise continuous, expanding transformation with a countable number of pieces can be approximated by the densities $\left\{f_{n}\right\}$, invariant under $\left\{\tau_{n}\right\}$, where $\tau_{n}$ is a finite approximation of $\tau$.

In $\S 3$, some examples are presented and an application to linear algebra is suggested. In $\S 4$, we discuss the application of the main result to first return maps of nonexpanding transformations.

\section{MAIN RESUlt}

Definition 1. Let $\tau_{n}, \tau$ be maps from $I=[0,1]$ into itself. We say $\tau_{n}$ converges to $\tau$ almost uniformly if given any $\varepsilon>0$ there exist a measurable set $A_{\varepsilon} \subset I, m(A)_{\varepsilon}>1-\varepsilon$, such that $\tau_{n} \rightarrow \tau$ uniformly on $A_{\varepsilon}$.

The following lemma will be useful in the sequel.

Lemma 1. Let $\tau_{n} \rightarrow \tau$ almost uniformly. Let $f_{n}$ be a fixed point of $P_{n}=P_{\tau_{n}}$. If $f_{n} \rightarrow f$ weakly in $L_{1}$, then $P_{\tau} f=f$.

Proof. We shall prove that the measures $f d m$ and $\left(P_{\tau} f\right) d m$ are equal. To do this it suffices to show that for any $g \in C(I): \int g\left(f-P f_{\tau}\right) d m=0$. We have

$$
\begin{aligned}
\left|\int g\left(f-P_{\tau} f\right) d m\right| \leq & \left|\int g\left(f-f_{n}\right) d m\right|+\left|\int g\left(f_{n}-P_{n} f_{n}\right) d m\right| \\
& +\left|\int g\left(P_{n} f_{n}-P_{\tau} f_{n}\right) d m\right|+\left|\int g\left(P_{\tau} f_{n}-P_{\tau} f\right) d m\right|
\end{aligned}
$$

The first term goes to 0 as $n \rightarrow \infty$ since $f_{n} \rightarrow f$ weakly in $L_{1}$. The second term is 0 since $P_{n} f_{n}=f_{n}$. Since

$$
\int g\left(P_{\tau} f_{n}-P_{\tau} f\right) d m=\int(g \circ \tau)\left(f_{n}-f\right) d m,
$$

and $g \circ \tau$ is bounded, using the continuity of $g$, the weak convergence of $f_{n}$ to $f$ implies that the fourth term goes to 0 as $n \rightarrow \infty$. It remains only to consider the third term. Since $f_{n} \rightarrow f$ weakly as $n \rightarrow \infty$, the sequence $\left\{f_{n}\right\}$ is uniformly integrable. Thus, given $\delta>0$ there exist $\varepsilon>0$ such that

$$
\int_{B}\left|f_{n}\right| d m<\delta
$$


for all $n \geq 1$, where $m(B)<\varepsilon$. Since $\tau_{n} \rightarrow \tau$ almost uniformly, $\tau_{n} \rightarrow \tau$ uniformly on a set $A_{\varepsilon}$, where $m\left(A_{\varepsilon}\right)>1-\varepsilon$. Returning to the third term of (1), we can write

$$
\begin{aligned}
& \left|\int g\left(P_{n} f_{n}-P_{\tau} f_{n}\right) d m\right| \\
& \quad \leq\left|\int_{A_{\varepsilon}}\left(g \circ \tau_{n}-g \circ \tau\right) f_{n} d m\right|+\left|\int_{A_{\varepsilon}^{c}}\left(g \circ \tau_{n}-g \circ \tau\right) f_{n} d m\right| \\
& \quad \leq \sup _{x \in A_{\varepsilon}}\left|g \circ \tau_{n}(x)-g \circ \tau(x)\right| \int\left|f_{n}\right| d m+2 \sup |g| \int_{A_{\varepsilon}^{c}}\left|f_{n}\right| d m \\
& \quad \leq \omega_{g}\left(\sup _{x \in A_{\varepsilon}} \tau_{n}(x)-\tau(x) \mid\right) \int\left|f_{n}\right| d m+2 \sup |g| \int_{A_{\varepsilon}^{c}}\left|f_{n}\right| d m
\end{aligned}
$$

where $\omega_{g}$ is the modulus of continuity of $g$. Since $\tau_{n} \rightarrow \tau$ uniformly on $A_{\varepsilon}$ and $\int\left|f_{n}\right| d m$ are uniformly bounded, the first term in (3) tends to 0 as $n \rightarrow \infty$. Since $m\left(A_{\varepsilon}^{c}\right) \leq \varepsilon$, it follows from (2) that $\int_{A_{\varepsilon}^{c}}\left|f_{n}\right| d m<\delta$, for all $n$. Hence, given any $\delta>0$

$$
\left|\int g\left(P_{n} f_{n}-P_{\tau} f_{n}\right) d m\right|<\omega_{g}\left(\sup _{x \in A_{\varepsilon}}\left|\tau_{n}(x)-\tau(x)\right|\right)+K \delta
$$

where $K=2 \sup |g|$. Therefore, the third term of (1) can be made arbitrarily small by the proper choice of $\varepsilon$ and $n, n \rightarrow \infty$, and the result is proved.

Definition 2. We say that the transformation $\tau: I \rightarrow I$ is countably piecewise expanding if (i) there exists a countable set $S \subset I$ such that for any connected component $J \subset I \backslash S, \tau_{\mid J}$ is a monotonic $C^{1}$-function satisfying: $\left|\tau^{\prime}\right| \geq \lambda>2$, (ii) we assume that $S$ has a finite number of limit points $L=\left\{s_{i}\right\}_{i=1}^{q}$, and that $V_{I} g=W<+\infty$, where

$$
g(x)= \begin{cases}0, & x \in S \\ \left|1 / \tau^{\prime}(x)\right|, & x \notin S\end{cases}
$$

Let $\tau$ be a countably piecewise expanding transformation. We shall now describe a procedure for constructing a sequence of transformations $\left\{\tau_{n}\right\}$ which approximate $\tau$. Let $\delta>0$ be smaller than $\left(\frac{1}{3} \min \left\{\left|s_{i}-s_{j}\right|: 1 \leq i, j \leq q\right.\right.$, $i \neq j\}$. For any point $s_{i}, i=1, \ldots, q$, we define one or two intervals $U_{i}(\delta)$, as follows: $U_{i}(\delta)=\left(s_{i}, s_{i}+\delta\right)$ if $s_{i}$ is a limit point of $S$ from the right; $U_{i}(\delta)=\left(s_{i}-\delta, s_{i}\right)$ if $s_{i}$ is a limit point of $S$ from the left, or both such intervals (with different indices) if $s_{i}$ is a limit point of $S$ from both sides.

Let $\varepsilon_{0}=\frac{1}{2}-1 / \lambda>0$. Consider the intervals $U_{i}(\delta), i=1, \ldots, \bar{q}$, where $\bar{q} \geq q$ since some of the limit points may be limit points from both sides. Let $H(\delta)=\bigcup_{i=1}^{q} U_{i}(\delta)$. Since $V_{l} g$ is finite, we can choose $\delta_{0}$ so small that $\sup _{H\left(\delta_{0}\right)} g<\varepsilon_{0} / 5$. Now let $\left\{\delta_{n}\right\}_{n=1}^{\infty}$ be any decreasing sequence of real numbers converging to $0, \delta_{1} \leq \delta_{0}$.

We define the approximation $\tau_{n}, n=1,2, \ldots$, to $\tau$ as follows: a) $\tau_{\left.n\right|_{\ell^{\prime},\left(\delta_{n}\right)}}$ is linear with $\left|\tau_{n}^{\prime}\right| \geq \lambda, i=1, \ldots, \bar{q}$; 
b) $\tau_{\left.n\right|_{\wedge M\left(\delta_{0}\right)}}$ is identical with $\tau$. We define

$$
g_{n}(x)= \begin{cases}0, & x \in S \backslash H\left(\delta_{0}\right) ; \\ 1 /\left|\tau_{n}^{\prime}\right|, & \text { elsewhere. }\end{cases}
$$

By the main result in [1], it follows that any $\tau_{n}$ has an absolutely continuous invariant measure $\mu_{n}$. Let $f_{n}$ be the density of this absolutely continuous invariant measure.

Theorem 1. The set $\left\{f_{n}\right\}_{n=1}^{\infty}$ has uniformly bounded variation and is therefore strongly compact in $L_{1}$.

We shall prove this theorem by means of the following lemma.

Lemma 2. There exists a partition $R$ of I into intervals such that for any $\mathscr{I} \in R$ and any $n=1,2, \ldots V_{\mathcal{f}} g_{n}<\frac{1}{2}$.

Proof. The jumps of $g$ do not exceed $\sup g$. Therefore, for any $\varepsilon>0$ and every $y \in I$ there exists some interval $E_{y}$ containing $y$, such that $V_{E_{y}} g<$ $\sup g+\varepsilon$. Since $I$ is compact, there exists a finite subcover of $\left\{E_{y}\right\}_{y \in I}$, call it $R_{1}$, such that for any $\mathscr{I} \in R_{1}: V_{\mathscr{I}} g<\sup g+\varepsilon$. Since $\sup g<1 / \lambda=\frac{1}{2}-\varepsilon_{0}$, putting $\varepsilon=\varepsilon_{0} / 5$ we get $V_{\mathscr{f}} g<\frac{1}{2}-4 \varepsilon_{0} / 5$, for any $\mathscr{I}$ from $R_{1}$.

We now refine $R_{1}$ a little as follows; let $R_{2}$ be the partition whose endpoints are $\left\{s_{1}, \ldots, s_{q}\right\} \cup\left\{\right.$ endpoints of intervals in $\left.R_{1}\right\}$. We now further refine $R_{2}$ by adding, if necessary, points close to $s_{1}, \ldots, s_{q}$ in such a way that the variation of $g$ over the resulting intervals adjacent to $s_{1}, \ldots, s_{q}$ is smaller than $2 \varepsilon_{0} / 5$. Call this new partition $R$.

Now $\sup g_{n}<1 / \lambda, \sup _{H\left(\delta_{n}\right)} g<\varepsilon_{0} / 5$ and $V_{\mathscr{G}} g<2 \varepsilon_{0} / 5$ for any $\mathscr{I} \in R$ such that $\mathscr{I} \cap H\left(\delta_{n}\right) \neq \varnothing$, for $n$ sufficiently large. Thus, we have for any $\mathscr{I} \in R, \mathscr{I} \cap H\left(\delta_{n}\right) \neq \varnothing$, and $n$ sufficiently large

$$
V_{\mathcal{F}} g_{n} \leq V_{\mathcal{F}} g+\sup _{\mathcal{I} \cap H\left(\delta_{n}\right)} g+\sup _{\mathcal{I} \cap H\left(\delta_{n}\right)} g_{n}(x)<\frac{3 \varepsilon_{0}}{5}+\frac{1}{\lambda}<\frac{1}{2} \text {. }
$$

Lemma 3. For any function $f$ of bounded variation, we have

$$
V_{I} P_{\tau_{n}} f \leq a V_{I} f+D\|f\|_{1},
$$

where

$$
a=\sup _{n}\left(\sup _{x \in I} g_{n}(x)+\max _{\mathcal{I} \in R} V_{\mathcal{F}} g_{n}\right) \leq \frac{1}{\lambda}+\frac{1}{2}<1
$$

and

$$
D=\sup _{n}\left(\max _{\mathscr{f} \in R}\left(V_{\mathscr{I}} g_{n} / m(J)\right)\right) \leq \frac{1}{2 \min _{\mathscr{f} \in R} m(J)}
$$

and $m$ is Lebesgue measure on $I$.

Proof. Corollary 3 of [1].

We are now ready to prove Theorem 1 . Let $f_{n}$ be an invariant density of $\tau_{n}$. The existence of $f_{n}$ follows from Lemma 3 by standard arguments. Again by Lemma 3, we obtain: $V_{I} f_{n}<a V_{I} f_{n}+D\left\|f_{n}\right\|_{1}$. Thus, $V_{I} f_{n} \leq D /(1-a)$, $n=1,2, \ldots$. This completes the proof of Theorem 1 . 
Theorem 2. If $f$ is a weak- $L_{1}$ limit point of the set of $\tau_{n}$-invariant densities $\left\{f_{n}\right\}_{n \geq 1}$, then $f$ is a $\tau$-invariant density.

Proof. A direct consequence of Lemma 1, Theorem 1, and the construction of $\tau_{n}$.

Corollary 1. If $\tau$ has a unique acim with density $f$, then the $\tau_{n}$-invariant densities $f_{n}$ converge to $f$ in $L_{1}$.

\section{EXAMPLES}

(1) It is well known that the Gauss transformation $\tau(x)=1 / x(\bmod 1)$ has invariant density $f(X)=(1 / \ln 2)(1 /(1+x))$. The transformation $\tau$ is countably piecewise expanding and Corollary 1 applies to it. The approximation transformations $\left\{\tau_{n}\right\}$ are defined as follows:

$$
\tau_{n}(x)= \begin{cases}\tau(x)(\bmod 1), & 1 / n<x \leq 1 \\ -n x+1, & 0 \leq x \leq 1 / n .\end{cases}
$$

The invariant density of $\tau_{n}$ can be computed by using a piecewise linear approximation to $\tau_{n}$, as is done in [4].

(2) Let $\tau$ be the piecewise linear, countably piecewise expanding transformation shown in Figure 1.

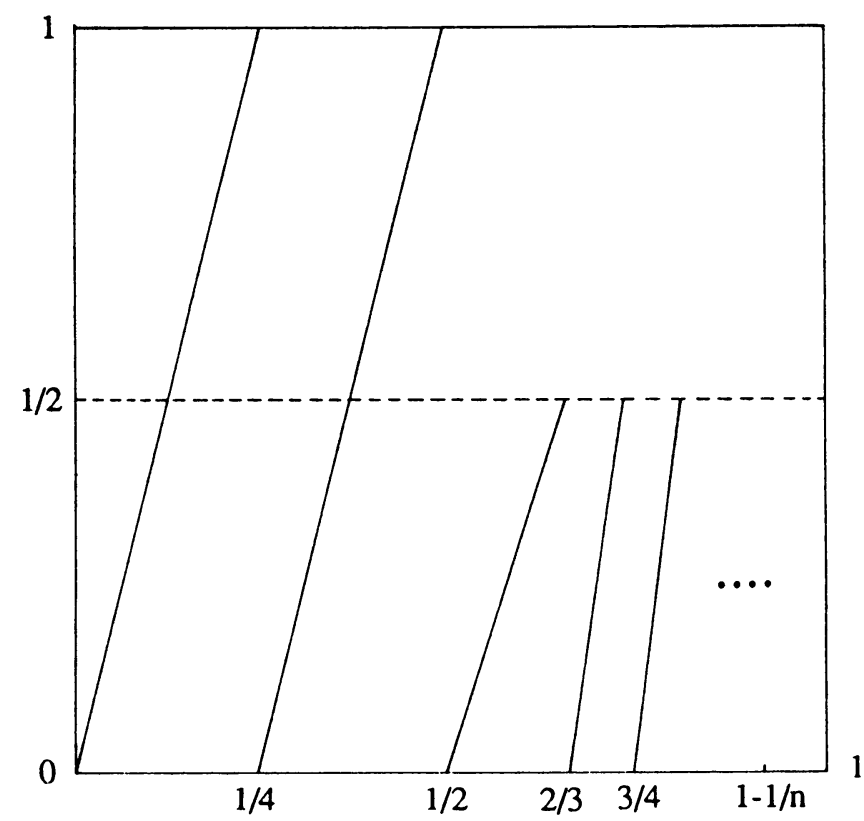

FIGURE 1. 
The Frobenius-Perron operator for $\tau$ can be represented by the matrix:

$$
M=\left[\begin{array}{cccccc}
\frac{1}{4} & \frac{1}{4} & \frac{1}{4} & \cdots & \frac{1}{4} & \cdots \\
\frac{1}{4} & \frac{1}{4} & \frac{1}{4} & \cdots & \frac{1}{4} & \cdots \\
\frac{1}{3} & 0 & 0 & \cdots & 0 & \cdots \\
\frac{1}{6} & 0 & 0 & \cdots & 0 & \cdots \\
\vdots & & & & & \\
\frac{2}{n(n+1)} & 0 & 0 & \cdots & 0 & \cdots \\
\vdots & & & & &
\end{array}\right] .
$$

Solving for the left eigenvector $\bar{\pi}=\left(\pi_{1}, \pi_{2}, \ldots\right)$, we obtain $\frac{1}{4} \pi_{1}+\frac{1}{4} \pi_{2}=\pi_{2}$, which implies that $\pi_{2}=\frac{1}{3} \pi_{1}$. Also, it is easy to see that $\pi_{n}=\frac{1}{3} \pi_{1}, n \geq 2$. Normalizing, we get:

$$
\frac{1}{4}\left(\pi_{1}+\pi_{2}\right)+\pi_{n} \sum_{n=2}^{\infty} \frac{1}{n(n+1)}=1 .
$$

On substituting for $\pi_{2}$ and $\pi_{n}$, we obtain $\pi_{1}=2$ and $\pi_{n}=\frac{2}{3}, n \geq 2$. Hence $\bar{\pi}=\left(2, \frac{2}{3}, \frac{2}{3}, \ldots\right)$ is the unique normalized invariant density of $\tau$. Let us now approximate $\tau$ by

$$
\tau_{N}(x)= \begin{cases}\tau(x), & 0 \leq x<1-1 / N \\ N x+1 /(2-N), & 1-1 / N \leq x \leq 1\end{cases}
$$

Then the invariant density of $\tau_{n}$ is the unique normalized left eigenvector of

$$
M_{n}=\left[\begin{array}{cccccc}
\frac{1}{4} & \frac{1}{4} & \frac{1}{4} & \cdots & \frac{1}{4} & \cdots \\
\frac{1}{4} & \frac{1}{4} & \frac{1}{4} & \cdots & \frac{1}{4} & \cdots \\
\frac{1}{3} & 0 & 0 & \cdots & 0 & \cdots \\
\frac{1}{6} & 0 & 0 & \cdots & 0 & \cdots \\
\vdots & & & & & \\
\frac{2}{N} & \frac{2}{N} & 0 & \cdots & 0 & \cdots
\end{array}\right] .
$$

By Corollary 1, the sequence of left eigenvectors $\left\{\bar{\pi}_{N}\right\}$ of $\left\{M_{N}\right\}$ converges coordinatewise to $\bar{\pi}$. We remark that Corollary 1 provides a general procedure for truncating certain non-negative infinite-dimensional matrices in such a way that the normalized left eigenvectors of the truncated matrices converge to the normalized left eigenvector of the infinite-dimensional matrix.

\section{First RETURN MAPS}

First return maps are often countably piecewise expanding. Consider, for example, $\tau: I \rightarrow I$, piecewise monotonic with a finite partition and having the property that at some fixed points $\left\{x_{i}\right\}_{i=1}^{s},\left|\tau^{\prime}\left(x_{i}\right)\right|=1$. Then $\tau$ is a nonexpanding map which has either a $\sigma$-finite or finite acim, but the approximation methods of $[3,4,5]$ do not apply. Let $W_{1}, \ldots, W_{s}$ be neighborhoods of $x_{1}, \ldots, x_{s}$. Then the first return map, $R_{W}$, of $\tau$ to the set $W=I \backslash \bigcup_{i=1}^{s} W_{i}$, is piecewise countable and expanding. $R_{W}$ can be approximated by the methods 
of $\S 2$. Thus there exists a sequence of piecewise monotonic expanding transformations $\left\{R_{n}\right\}_{n \geq 1}$, each having a finite partition, such that the associated densities $\left\{g_{n}\right\}_{n \geq 1}$ converge to $g$, the invariant density of $R_{W}$.

Let $\mu_{W}$ be the measure induced by $g$. Then it is easy to show (Lemma 2 of [2]) that for any set $D \subset W, \mu_{W}(D)=\mu_{\left.\right|^{*}}(D)$, where $\mu_{\left.\right|_{W}}$ is the $\sigma$-finite acim of $\tau$ restricted to $W$ and normalized on $W$. Hence the approximating densities $\left\{g_{n}\right\}_{n \geq 1}$, restricted to $W$, also approximate the density of $\mu_{\left.\right|_{w}}$.

Consider the following example [8]:

$$
\tau(x)= \begin{cases}\frac{x}{1-x}=\tau_{1}(x), & 0 \leq x \leq \frac{1}{2} \\ 2 x-1=\tau_{2}(x), & \frac{1}{2} \leq x \leq 1 .\end{cases}
$$

Since $\tau^{\prime}(0)=1$, the first return map to the set $G=\left(\frac{1}{2}, 1\right)$, say, has a countable number of pieces:

$$
R_{G}(x)= \begin{cases}\tau(x), & x \in\left(x_{0}, 1\right) \\ \tau^{n+1}(x), & x \in\left(x_{n}, x_{n+1}\right), n \geq 1,\end{cases}
$$

where $\left\{x_{i}\right\} \subset G$ and $\left\{y_{i}\right\} \subset\left[0, \frac{1}{2}\right]$ are defined by

$$
y_{0}=\frac{1}{2}, \quad y_{n+1}=\tau_{1}^{-1}\left(y_{n}\right), \quad \text { and } x_{n}=\tau_{2}^{-1}\left(y_{n}\right), \quad n \geq 1 .
$$

$R_{G}$ can be approximated by the procedure of $\S 2$ and the measures of the resulting densities also approximate the normalized measure $\mu_{\left.\right|_{G}}$, where $\mu$ is the $\sigma$-finite measure invariant under $\tau$ on the set $G$.

Remark. An alternate method for approximating the first return map of a nonexpanding map is presented in [6].

\section{REFERENCES}

1. M. Rychlik, Bounded variation and invariant measures, Studia Math., LXXVI (1983), 69-80.

2. G. Pianigiani, First return map and invariant measures, Israel J. Math. 35 (1980), 32-48.

3. G. Keller, Stochastic stability in some chaotic dynamical systems, Monatsh. Math. 94 (1982), 313-333.

4. P. Góra and A. Boyarsky, Compactness and invariant densities for families of expanding, piecewise monotonic transformations, preprint.

5. T-Y Li, Finite approximation for the Frobenius-Perron operator. A solution to Ulam's conjecture, J. Approx. Theory 17 (1976), 177-186.

6. A. Boyarsky, Approximating the $\sigma$-finite measure invariant under a non-expanding map, J. Math. Anal. Appl. 78 (1980), 222-232.

7. P. Góra and A. Boyarsky, Why computers like Lebesgue measure, Applic. 16, No. 4, 321-329, 1988. Comput. Math.

8. A. Lasota and J. A. Yorke, , On the existence of invariant measures for piecewise monotonic transformations, Trans. Amer. Math. Soc. 186 (1973), 481-488.

Department of Mathematics, Warsaw University, Warsaw, Poland

Department of Mathematics, Concordia University, 7141 Sherbrooke St. West, Montreal, Canada H4B 1 R6 